\title{
Overcoming the Challenges of Megabase-Sized Plasmid Construction in Escherichia coli
}

Takahito Mukai, ${ }^{*}, 1$ Tatsuya Yoneji, ${ }^{1}$ Kayoko Yamada, Hironobu Fujita, Seia Nara and Masayuki Su'etsugu*

Department of Life Science, College of Science, Rikkyo University, 3-34-1 Nishi-lkebukuro, Toshima-ku, Tokyo 171-8501, Japan

\section{Supplementary}

Table S1. The sequences of the genetic cassettes used in this study.

>TrrnC-zeo-PEM7-oriT>-BGHfwd-Cpf1s1

GACGCAGTTCAACTCAGCTGTACACATCAAAGCTTTCGACGAAATGCATGcaaaaaaatccttagctttcgctaag gatctgcaTCAGTCCTGCTCCTCGGCCACGAAGTGCACGCAGTTGCCGGCCGGGTCGCGCAGGGCGAACTCCCGCCC CCACGGCTGCTCGCCGATCTCGGTCATGGCCGGCCCGGAGGCGTCCCGGAAGTTCGTGGACACGACCTCCGACCACT CGGCGTACAGCTCGTCCAGGCCGCGCACCCACACCCAGGCCAGGGTGTTGTCCGGCACCACCTGGTCCTGGACCGCG CTGATGAACAGGGTCACGTCGTCCCGGACCACACCGGCGAAGTCGTCCTCCACGAAGTCCCGGGAGAACCCGAGCCG GTCGGTCCAGAACTCGACCGCTCCGGCGACGTCGCGCGCGGTGAGCACCGGAACGGCACTGGTCAACTTGGCCATGG TTTAGTTCCTCACCTTGTCGTATTATACTATGCCGATATACTATGCCGATGATTAATTGTCAACACGTGCTGATCAG ATCCGCAGCGCTTTTCCGCTGCATAACCCTGCTTCGGGGTCATTATAGCGATTTTTTCGGTATATCCATCCTTTTTC GCACGATATACAGGATTTTGCCAAAGGGTTCGTGTAGACTTTCCTTGgtgtatccaacggcgtcagccgggcaggat aggtgaagtaggcccacccgcgagcgggtgttccttcttcactgtccettattcGCACCTGGCGGTGCTCAACGGGA ATCCTGCTCTGCGAGGCTGGCCGgCTGgCAACTAGAAGGCACAGCCTTTAGAGAAGTCATTTAATAAGGCCACT

>TrrnC-spec-PEM7-oriT>-BGHfwd-Cpf1s1

GACGCAGTTCAACTCAGCTGTACACATCAAAGCTTTCGACGAAATGCATGcaaaaaaatccttagctttcgctaag gatctgcaTTATTTGCCGACTACCTTGGTGATCTCGCCTTTCACGTAGTGGACAAATTCTTCCAACTGATCTGCGCG CGAGGCCAAGCGATCTTCTTCTTGTCCAAGATAAGCCTGTCTAGCTTCAAGTATGACGGGCTGATACTGGGCCGGCA GGCGCTCCATTGCCCAGTCGGCAGCGACATCCTTCGGCGCGATTTTGCCGGTTACTGCGCTGTACCAAATGCGGGAC AACGTAAGCACTACATTTCGCTCATCGCCAGCCCAGTCGGGCGGCGAGTTCCATAGCGTTAAGGTTTCATTTAGCGC CTCAAATAGATCCTGTTCAGGAACCGGATCAAAGAGTTCCTCCGCCGCTGGACCTACCAAGGCAACGCTATGTTCTC TTGCTTTtGTCAGCAAGATAGCCAGATCAATGTCGATCGTGGCTGGCTCGAAGATACCTGCAAGAATGTCATTGCGC TGCCATTCTCCAAATTGCAGTTCGCGCTTAGCTGGATAACGCCACGGAATGATGTCGTCGTGCACAACAATGGTGAC 
TTCTACAGCGCGGAGAATCTCGCTCTCTCCAGGGGAAGCCGAAGTTTCCAAAAGGTCGTTGATCAAAGCTCGCCGCG TTGTTTCATCAAGCCTTACGGTCACCGTAACCAGCAAATCAATATCACTGTGTGGCTTCAGGCCGCCATCCACTGCG GAGCCGTACAAATGTACGGCCAGCAACGTCGGTTCGAGATGGCGCTCGATGACGCCAACTACCTCTGATAGTTGAGT CGATACTTCGGCGATCACCGCTTCCCTCATGGTTTAGTTCCTCACCTTGTCGTATTATACTATGCCGATATACTATG CCGATGATTAATTGTCAACACGTGCTGATCAGATCCGCAGCGCTTTTCCGCTGCATAACCCTGCTTCGGGGTCATTA TAGCGATTTTTTCGGTATATCCATCCTTTTTCGCACGATATACAGGATTTTGCCAAAGGGTTCGTGTAGACTTTCCT TGgtgtatccaacggcgtcagccgggcaggataggtgaagtaggcccacccgcgagcgggtgttccttcttcactgt cccttattcGCACCTGGCGGTGCTCAACGGGAATCCTGCTCTGCGAGGCTGGCCGGCTGGCAACTAGAAGGCACAGC CTTTAGAGAAGTCATTTAATAAGGCCACT

>Cpf1s3-attP-Tlpp-spec-PEM7-oriT>-BGHfwd

CTACTAATACAAAAGGCTCTAGACCAAAGGTCACTAATACTATCTAAGTAGTTGATTCATAGTGACTGGATATGTTG CGTTTTGTCGCATTATGTAGTCTATCATTTAACCACAGATTAGTGTAATGCGATGATTTTTAAGTGATTAATGTTAT TTTGTCATCCTTTAGGTGAATAAGTTGTATATTTAAAATCTCTTTAATTATCAGTAAATTAATGTAAGTAGGTCATT ATTAGTCAAAATAAAATCATTTGCaaaaaaatggcgcacaatgtgcgcCaTTATTTGCCGACTACCTTGGTGATCT CGCCTTTCACGTAGTGGACAAATTCTTCCAACTGATCTGCGCGCGAGGCCAAGCGATCTTCTTCTTGTCCAAGATAA GCCTGTCTAGCTTCAAGTATGACGGGCTGATACTGGGCCGGCAGGCGCTCCATTGCCCAGTCGGCAGCGACATCCTT CGGCGCGATTTTGCCGGTTACTGCGCTGTACCAAATGCGGGACAACGTAAGCACTACATTTCGCTCATCGCCAGCCC AGTCGGGCGGCGAGTTCCATAGCGTTAAGGTTTCATTTAGCGCCTCAAATAGATCCTGTTCAGGAACCGGATCAAAG AGTTCCTCCGCCGCTGGACCTACCAAGGCAACGCTATGTTCTCTTGCTTTTGTCAGCAAGATAGCCAGATCAATGTC GATCGTGGCTGGCTCGAAGATACCTGCAAGAATGTCATTGCGCTGCCATTCTCCAAATTGCAGTTCGCGCTTAGCTG GATAACGCCACGGAATGATGTCGTCGTGCACAACAATGGTGACTTCTACAGCGCGGAGAATCTCGCTCTCTCCAGGG GAAGCCGAAGTTTCCAAAAGGTCGTTGATCAAAGCTCGCCGCGTTGTTTCATCAAGCCTTACGGTCACCGTAACCAG CAAATCAATATCACTGTGTGGCTTCAGGCCGCCATCCACTGCGGAGCCGTACAAATGTACGGCCAGCAACGTCGGTT CGAGATGGCGCTCGATGACGCCAACTACCTCTGATAGTTGAGTCGATACTTCGGCGATCACCGCTTCCCTCATGGTT TAGTTCCTCACCTTGTCGTATTATACTATGCCGATATACTATGCCGATGATTAATTGTCAACACGTGCTGATCAGAT CCGCAGCGCTTTTCCGCTGCATAACCCTGCTTCGGGGTCATTATAGCGATTTTTTCGGTATATCCATCCTTTTTCGC ACGATATACAGGATTTTGCCAAAGGGTTCGTGTAGACTTTCCTTGgtgtatccaacggcgtcagccgggcaggatag gtgaagtaggcccacccgcgagcgggtgttccttcttcactgtcccttattcGCACCTGGCGGTGCTCAACGGGAAT CCTGCTCTGCGAGGCTGGCCGGCTGGCAACTAGAAGGCACAG

>Cpf1s3-attP-Tlpp-zeo-PEM7-oriT>-BGHfwd CTACTAATACAAAAGGCTCTAGACCAAAGGTCACTAATACTATCTAAGTAGTTGATTCATAGTGACTGGATATGTTG CGTTTTGTCGCATTATGTAGTCTATCATTTAACCACAGATTAGTGTAATGCGATGATTTTTAAGTGATTAATGTTAT TTTGTCATCCTTTAGGTGAATAAGTTGTATATTTAAAATCTCTTTAATTATCAGTAAATTAATGTAAGTAGGTCATT ATTAGTCAAAATAAAATCATTTGcaaaaaaatggcgcacaatgtgcgcCaTTATCAGTCCTGCTCCTCGGCCACGA AGTGCACGCAGTTGCCGGCCGGGTCGCGCAGGGCGAACTCCCGCCCCCACGGCTGCTCGCCGATCTCGGTCATGGCC GGCCCGGAGGCGTCCCGGAAGTTCGTGGACACGACCTCCGACCACTCGGCGTACAGCTCGTCCAGGCCGCGCACCCA 
CACCCAGGCCAGGGTGTTGTCCGGCACCACCTGGTCCTGGACCGCGCTGATGAACAGGGTCACGTCGTCCCGGACCA CACCGGCGAAGTCGTCCTCCACGAAGTCCCGGGAGAACCCGAGCCGGTCGGTCCAGAACTCGACCGCTCCGGCGACG TCGCGCGCGGTGAGCACCGGAACGGCACTGGTCAACTTGGCCATGGTTTAGTTCCTCACCTTGTCGTATTATACTAT GCCGATATACTATGCCGATGATTAATTGTCAACACGTGCTGATCAGATCCGCAGCGCTTTTCCGCTGCATAACCCTG CTTCGGGGTCATTATAGCGATTTTTTCGGTATATCCATCCTTTTTCGCACGATATACAGGATTTTGCCAAAGGGTTC GTGTAGACTTTCCTTGgtgtatccaacggcgtcagccgggcaggataggtgaagtaggcccacccgcgagcgggtgt tccttcttcactgtccettattcGCACCTGGCGGTGCTCAACGGGAATCCTGCTCTGCGAGGCTGGCCGGCTGGCAA CTAGAAGGCACAG

>Cpf1s3-attPv-Tlpp-zeo-PEM7-oriT>-BGHfwd

CTACTAATACAAAAGGCTCTAGACCAAAGGTCACTAATACTATCTAAGTAGTTGATTCATAGTGACTGGATATGTTG CGTTTTGTCGCATTATGTAGTCTATCATTTAACCACAGATTAGTGTAATGCGATGATTTTTAAGTGATTAATGTTAT TTTGTCATCCTTTTACAATGTAAGTTGTATATTTAAAATCTCTTTAATTATCAGTAAATTAATGTAAGTAGGTCATT ATTAGTCAAAATAAAATCATTTGCaaaaaaatggcgcacaatgtgcgccaTTATCAGTCCTGCTCCTCGGCCACGA AGTGCACGCAGTTGCCGGCCGGGTCGCGCAGGGCGAACTCCCGCCCCCACGGCTGCTCGCCGATCTCGGTCATGGCC GGCCCGGAGGCGTCCCGGAAGTTCGTGGACACGACCTCCGACCACTCGGCGTACAGCTCGTCCAGGCCGCGCACCCA CACCCAGGCCAGGGTGTTGTCCGGCACCACCTGGTCCTGGACCGCGCTGATGAACAGGGTCACGTCGTCCCGGACCA CACCGGCGAAGTCGTCCTCCACGAAGTCCCGGGAGAACCCGAGCCGGTCGGTCCAGAACTCGACCGCTCCGGCGACG TCGCGCGCGGTGAGCACCGGAACGGCACTGGTCAACTTGGCCATGGTTTAGTTCCTCACCTTGTCGTATTATACTAT GCCGATATACTATGCCGATGATTAATTGTCAACACGTGCTGATCAGATCCGCAGCGCTTTTCCGCTGCATAACCCTG CTTCGGGGTCATTATAGCGATTTTTTCGGTATATCCATCCTTTTTCGCACGATATACAGGATTTTGCCAAAGGGTTC GTGTAGACTTTCCTTGgtgtatccaacggcgtcagccgggcaggataggtgaagtaggcccaccegcgagcgggtgt tccttcttcactgtccettattcGCACCTGGCGGTGCTCAACGGGAATCCTGCTCTGCGAGGCTGGCCGGCTGGCAA CTAGAAGGCACAG

$>$ Pkan'-hyg-Pcat'-cat5' - FRT-Cpf1s1

cgctgcttttgcacgtgagaaagccacgttgtgtctcaaaatctctgatgttgcattgcacaagataaaatatatc atcatgaacaataaactgtctgcttacataaacggaaaattttttttcaaaagtacttgacaacataacggataac aattGtaatagattcaattgtaacgtaatacaaggaacgtgttATGAAAAAACCGGAACTGACCGCAACCAGCGTTG AAAAATTTCTGATCGAAAAATTCGATAGCGTGAGCGATCTGATGCAGCTGAGCGAAGGTGAAGAAAGCCGTGCATTT AGCTTTGATGTTGGTGGTCGTGGTTATGTTCTGCGTGTTAATAGCTGTGCAGATGGCTTCTATAAAGATCGTTATGT GTATCGTCATTTTGCAAGCGCAGCACTGCCGATTCCGGAAGTTCTGGATATTGGTGAATTTAGCGAAAGCCTGACCT ATTGTATTAGCCGTCGTGCACAGGGTGTTACCCTGCAGGATCTGCCGGAAACCGAACTGCCTGCAGTTCTGCAGCCG GTTGCCGAAGCAATGGATGCAATTGCCGCAGCAGATCTGAGCCAGACCAGCGGTTTTGGTCCGTTTGGTCCTCAAGG TATTGGTCAGTATACCACCTGGCGTGATTTTATCTGTGCCATTGCAGATCCGCATGTTTATCATTGGCAGACCGTTA TGGATGATACCGTTAGCGCAAGCGTTGCACAGGCACTGGATGAACTGATGCTGTGGGCAGAAGATTGTCCTGAAGTT CGTCATCTGGTTCATGCAGATTTTGGTAGCAATAATGTGCTGACCGATAATGGTCGTATTACCGCAGTTATTGATTG GAGCGAAGCCATGTTTGGTGATAGCCAGTATGAAGTTGCCAACATCTTTTTTTGGCGTCCGTGGCTGGCATGTATGG 
AACAGCAGACCCGTTATTTTGAACGTCGTCATCCGGAACTGGCAGGTAGTCCGCGTCTGCGTGCCTATATGCTGCGT ATTGGTCTGGATCAGCTGTATCAGTCACTGGTTGATGGTAATTTTGATGATGCAGCATGGGCACAGGGTCGTTGTGA TGCCATTGTTCGTAGCGGTGCAGGCACCGTTGGTCGTACCCAGATTGCACGTCGTAGCGCAGCAGTTTGGACCGATG GTTGTGTTGAAGTGCTGGCAGATAGCGGTAATCGTCGTCCGAGCACACGTCCGGATCGTGAAATGGGTGAAGCAAAT TAAtgCGCTGGCAACTAGAAGGCACAGcgtaagaggttccaactttcaccatattgacataagatcactaccgggcg tataatttgagttatcgagattttcaggagctaaggaagctaaaATGGAGAAAAAAATCACTGGATATACCACCGTT GATATATCCCAATGGCATCGTAAAGAACATTTTGAGGCATTTCAGTCAGTTGCTCAATGTACCTATAACCAGACCGT TCAGCTGGATATTACGGCCTTTTTAAAGACCGTAAAGAAAAATAAGCACAAGTTTTATCCGGCCTTTATTCACATTC TTGCCCGCCTGATGAATGCTCATCCGGAATTCCGTATGGCAATGAAAGACGGTGAGCTGGTGATATGGGATAGTGTT CACCCTTGTTACACCGTTTTCCATGAGCAAACTGAAACGTTTTCATCGCTCTGGAGTGAATACCACGACGATTTCCG GCAGTTTCTACACATATATTCGCAAGATGTGGCGTGTTACggaagttcctattctctagaagtataggaacttcCA GGTCTTTCTCAAGCCGACCtttagagaagtcatttaataaggccact

>Cpf1s3-attP-FRT-cat3'-Ttrp-PEM7-zeo-Tlpp-TrrnC

CTACTAATACAAAAGGCTCTAGACCAAAGGTCACTAATACTATCTAAGTAGTTGATTCATAGTGACTGGATATGTTG CGTTTTGTCGCATTATGTAGTCTATCATTTAACCACAGATTAGTGTAATGCGATGATTTTTAAGTGATTAATGTTAT

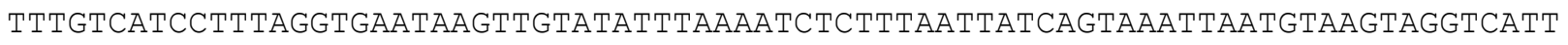
ATTAGTCAAAATAAAATCATTTGgctgcttttgcacgtgagGAAGTTCCTATTCTCTAGAAAGTATAGGAACTTCt GAAAACCTGGCCTATTTCCCTAAAGGGTTTATTGAGAATATGTTTTTCGTCTCAGCCAATCCCTGGGTGAGTTTCAC CAgTTTTGAтTAAACGTGGCCAATATGGACAACTTCTTCGCCCCCGTTTTCACCATGGGCAAATATTATACGCAAG GCGACAAGGTGCTGATGCCGCTGGCGATTCAGGTTCATCATGCCGTtTGTGATGGCTTCCATGTCGGCAGAATGCTT AATGAATTACAACAGTACTGCGATGAGTGGCAGGGCGGGGCGTAAtcccacagccgccagttccgctggcggcattt tgACTGCCATGGAAAATCGATGTTCTTCGGATCTGATCAGCACGTGTTGACAATTAATCATCGGCATAGTATATCGG CATAGTATAATACGACAAGGTGAGGAACTAAACCATGGCCAAGTTGACCAGTGCCGTTCCGGTGCTCACCGCGCGCG ACGTCGCCGGAGCGGTCGAGTTCTGGACCGACCGGCTCGGGTTCTCCCGGGACTTCGTGGAGGACGACTTCGCCGGT GTGGTCCGGGACGACGTGACCCTGTTCATCAGCGCGGTCCAGGACCAGGTGGTGCCGGACAACACCCTGGCCTGGGT GTGGGTGCGCGGCCTGGACGAGCTGTACGCCGAGTGGTCGGAGGTCGTGTCCACGAACTTCCGGGACGCCTCCGGGC CGGCCATGACCGAGATCGGCGAGCAGCCGTGGGGGCGGGAGTTCGCCCTGCGCGACCCGGCCGGCAACTGCGTGCAC TTCGTGGCCGAGGAGCAGGACTGAtccgaaaatggcgcacattgtgcgccattttttttgGATCTGCGTTTCCTCA AACAGTTTAAATAAtgcagatccttagcgaaagctaaggatttttttg

>PEM7-zeo-pheS (T251S-A294G) -TrrnC

CGGATCTGATCAGCACGTGTTGACAATTAATCATCGGCATAGTATATCGGCATAGTATAATACGACAAGGTGAGGAA CTAAACCATGGCCAAGTTGACCAGTGCCGTTCCGGTGCTCACCGCGCGCGACGTCGCCGGAGCGGTCGAGTTCTGGA CCGACCGGCTCGGGTTCTCCCGGGACTTCGTGGAGGACGACTTCGCCGGTGTGGTCCGGGACGACGTGACCCTGTTC ATCAGCGCGGTCCAGGACCAGGTGGTGCCGGACAACACCCTGGCCTGGGTGTGGGTGCGCGGCCTGGACGAGCTGTA CGCCGAGTGGTCGGAGGTCGTGTCCACGAACTTCCGGGACGCCTCCGGGCCGGCCATGACCGAGATCGGCGAGCAGC CGTGGGGGCGGGAGTTCGCCCTGCGCGACCCGGCCGGCAACTGCGTGCACTTCGTGGCCGAGGAGCAGGACTGAtga 
ggaaaccATGTCACATCTCGCAGAACTGGTTGCCAGTGCGAAGGCGGCCATTAGCCAGGCGTCAGATGTTGCCGCG TTAGATAATGTGCGCGTCGAATATTTGGGTAAAAAAGGGCACTTAACCCTTCAGATGACGACCCTGCGTGAGCTGCC GCCAGAAGAGCGTCCGGCAGCTGGTGCGGTTATCAACGAAGCGAAAGAGCAGGTTCAGCAGGCGCTGAATGCGCGTA AAGCGGAACTGGAAAGCGCTGCACTGAATGCGCGTCTGGCGGCGGAAACGATTGATGTCTCTCTGCCAGGTCGTCGC ATTGAAAACGGCGGTCTGCATCCGGTTACCCGTACCATCGACCGTATCGAAAGTTTCTTCGGTGAGCTTGGCTTTAC CGTGGCAACCGGGCCGGAAATCGAAGACGATTATCATAACTTCGATGCTCTGAACATTCCTGGTCACCACCCGGCGC GCGCTGACCACGACACTTTCTGGTTTGACACTACCCGCCTGCTGCGTACCCAGACCTCTGGCGTACAGATCCGCACC ATGAAAGCCCAGCAGCCACCGATTCGTATCATCGCGCCTGGCCGTGTTTATCGTAACGACTACGACCAGACTCACAC GCCGATGTTCCATCAGATGGAAGGTCTGATTGTTGATACCAACATCAGCTTTACCAACCTGAAAGGCACGCTGCACG АСTTCCTGCGTAACTTCTTTGAGGAAGATTTGCAGATTCGCTTCCGTCCTTCCTACTTCCCGTTTTCCGAACCTTCT GCAGAAGTGGACGTCATGGGTAAAAACGGTAAATGGCTGGAAGTGCTGGGCTGCGGGATGGTGCATCCGAACGTGTT GCGTAACGTTGGCATCGACCCGGAAGTTTACTCTGGTTTCGGCTTCGGGATGGGGATGGAGCGTCTGACTATGTTGC GTTACGGCGTCACCGACCTGCGTTCATTCTTCGAAAACGATCTGCGTTTCCTCAAACAGTTTAAATAAtgcagatcC ttagcgaaagctaaggatttttttg

>tet-PEM7-oriT>-BGHfwd

TTAGGTCGAGGTGGCCCGGCTCCATGCACCGCGACGCAACGCGGGGAGGCAGACAAGGTATAGGGCGGCGCCTACAA TCCATGCCAACCCGTTCCATGTGCTCGCCGAGGCGGCATAAATCGCCGTGACGATCAGCGGTCCAaTGATCGAAGTT AgGCTGGTAAGAGCCGCGAGCGATCCTTGAAGCTGTCCCTGATGGTCGTCATCTACCTGCCTGGACAGCATGGCCTG CAACGCGGGCATCCCGATGCCGCCGGAAGCGAGAAGAATCATAATGGGGAAGGCCATCCAGCCTCGCGTCGCGAACG CCAGCAAGACGTAGCCCAGCGCGTCGGCCGCCATGCCGGCGATAATGGCCTGCTTCTCGCCGAAACGTTTGGTGGCG GGACCAGTGACGAAGGCTTGAGCGAGGGCGTGCAAGATTCCGAATACCGCAAGCGACAGGCCGATCATCGTCGCGCT CCAGCGAAAGCGGTCCTCGCCGAAAATGACCCAGAGCGCTGCCGGCACCTGTCCTACGAGTTGCATGATAAAGAAGA CAGTCATAAGTGCGGCGACGATAGTCATGCCCCGCGCCCACCGGAAGGAGCTGACTGGGTTGAAGGCTCTCAAGGGC AtCGgtCGACGCTCTCCCTTATGCGACTCCTGCATtAgGAAGCAGCCCAGTAGTAGGTtGAgGCCGTTGAGCACCGC CGCCGCAAGGAATGGTGCATGCAAGGAGATGGCGCCCAACAGTCCCCCGGCCACGGGGCCTGCCACCATACCCACGC CGAAACAAGCGCTCATGAGCCCGAAGTGGCGAGCCCGATCTTCCCCATCGGTGATGTCGGCGATATAGGCGCCAGCA ACCGCACCTGTGGCGCCGGTGATGCCGGCCACGATGCGTCCGGCGTAGAGGATCCACAGGACGGGTGTGGTCGCCAT GATCGCGTAGTCGATAGTGGCTCCAAGTAGCGAAGCGAGCAGGACTGGGCGGCGGCCAAAGCGGTCGGACAGTGCTC CGAGAACGGGTGCGCATAGAAATTGCATCAACGCATATAGCGCTAGCAGCACGCCATAGTGACTGGCGATGCTGTCG GAATGGACGATATCCCGCAAGAGGCCCGGCAGTACCGGCATAACCAAGCCTATGCCTACAGCATCCAGGGTGACGGT GCCGAGGATGACGATGAGCGCATTGTTAGATTTCATGGTTTAGTTCCTCACCTTGTCGTATTATACTATGCCGATAT ACTATGCCGATGATTAATTGTAACACGTGCTGATCAGATCCGCAGCGCTTTTCCGCTGCATAACCCTGCTTCGGGG

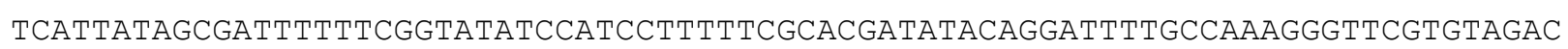
TTTCCTTGgtgtatccaacggcgtcagccgggcaggataggtgaagtaggcccacccgcgagcgggtgttccttctt cactgtcccttattcGCACCTGGCGGTGCTCAACGGGAATCCTGCTCTGCGAGGCTGGCCGGCTGGCAACTAGAAGG CACAG 
>Pkan'-gent (pseudogene)-gent (pFastBAC1)-TrrnC-RCRori

AAAGCCACGTTGTGTCTCAAAATCTCTGATGTTGCATTGCACAAGATAAAAATATATCATCATGAACAATAAAACTG TCTGCTTACATAAACGGAAAATTTTTTTCAAAAGTACTTGACAACATAACGGATAACAATTATAATAGATTCAATT GTAACGTAATACAAGGAACGTGTTATGTTACGCAGCAGCAACGATGTTACGCAGCAGGGTAGCCGTCCGAAAACCAA ATtAGgTGGTAGCAGCATGGGTATtATTCGTACCTGTCGTCTGGGTCCAGATCAGGTTAAAAGCATGCGTGCAGCAC TGGACCTGTTTGGTCGTGATTTGGTGATGTTGCAACCTATAGCCAGCATCAGCCGGATAGCGATTATCTGGGTAAT CTGCTGCGTTCCAAAACCTTTATGCACTGGCAGCATtTGATCAAGAAGCAGTTGTTGGAGCCCTGGCAGCCTATGT TCTGCCTAAGTTTGAACAGCCTCGTAGCGAAATCTATATCTATGATCTGGCAGTTAGCGGTGAACATCGTCGTCAGG GTATTGCAACCGCACTGATTAACCTGCTGAAACATGAAGCAAATGCACTGGGTGCCTATGTTATTTATGTTCAGGCA GATTATGGTGATGATCCGGCAGTGGCACTGTATACCAAACTGGGTATTCGTGAAGAAGTGATGCACTTTGATATTGA TCCGAGCACCGCAACCTAAGACGCACACCGTGGAAACGGATGAAGGCACGAACCCAGTTGACATAAGCCTGTTCGGT TCGTAAACTGTAATGCAAGTAGCGTATGCGCTCACGCAACTGGTCCAGAACCTTGACCGAACGCAGCGGTGGTAACG GCGCAGTGGCGGTTTCATGGCTTGTtATGACTGTTTTTTGTACAGTCTATGCCTCGGGCATCCAAGCAGCAAGCG CGTTACGCCGTGGGTCGATGTTTGATGTTATGGAGCAGCAACGATGTTACGCAGCAGCAACGATGTTACGCAGCAGG GCAGTCGCCCTAAAACAAAGTTAGGTGGCTCAAGTATGGGCATCATTCGCACATGTAGGCTCGGCCCTGACCAAGTC AAATCCATGCGGGCTGCTCTTGATCTTTTCGGTCGTGAGTTCGGAGACGTAGCCACCTACTCCCAACATCAGCCGGA CTCCGATTACCTCGGGAACTTGCTCCGTAGTAAGACATTCATCGCGCTTGCTGCCTTCGACCAAGAAGCGGTTGTTG GCGCTCTCGCGGCTTACGTTCTGCCCAAGTTTGAGCAGCCGCGTAGTGAGATCTATATCTATGATCTCGCAGTCTCC GGCGAGCACCGGAGGCAGGGCATTGCCACCGCGCTCATCAATCTCCTCAAGCATGAGGCCAACGCGCTTGGTGCTTA TGTGATCTACGTGCAAGCAGATTACGGTGACGATCCCGCAGTGGCTCTCTATACAAAGTTGGGCATACGGGAAGAAG TGATGCACTTTGATATCGACCCAAGTACCGCCACCTAATGCAGATCCTTAGCGAAAGCTAAGGATTTTTTTTGCCTC GACGGAAATTCTTGTCGGCTTGAGAAAGACCTGAGTTATCCACAAAGTTATGCACTTGCAAGAGGGTCATTTTCACA

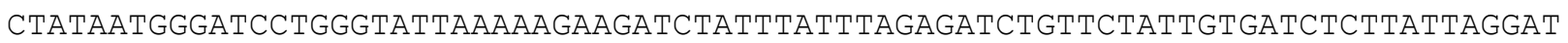
CGCACTGCCCTGTGGATAACAAGGATCCGGCTTTTAAGATCAACAACCTGGAAAGGATCATTAACTGTGAATGATCG GTGATCCTGGACCGTATAAGCTGGGATCAGAATGAGGGGTTATACACAACTCAAAAACTGAACAACAGTTGTTCTTT GGATAACTACCGGTTGATCCAAGCTTCCTGACAGAGTTATCCACAGTAGATCGCACGATCTGTCAGCTCATTTCAGA

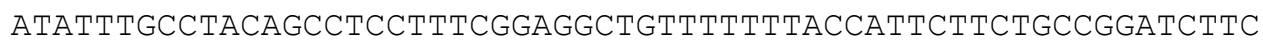

\section{Table S2. The modifications to the genome sequence of HST08 to develop HST08 $\Delta$ attB $\Delta$ tus.}

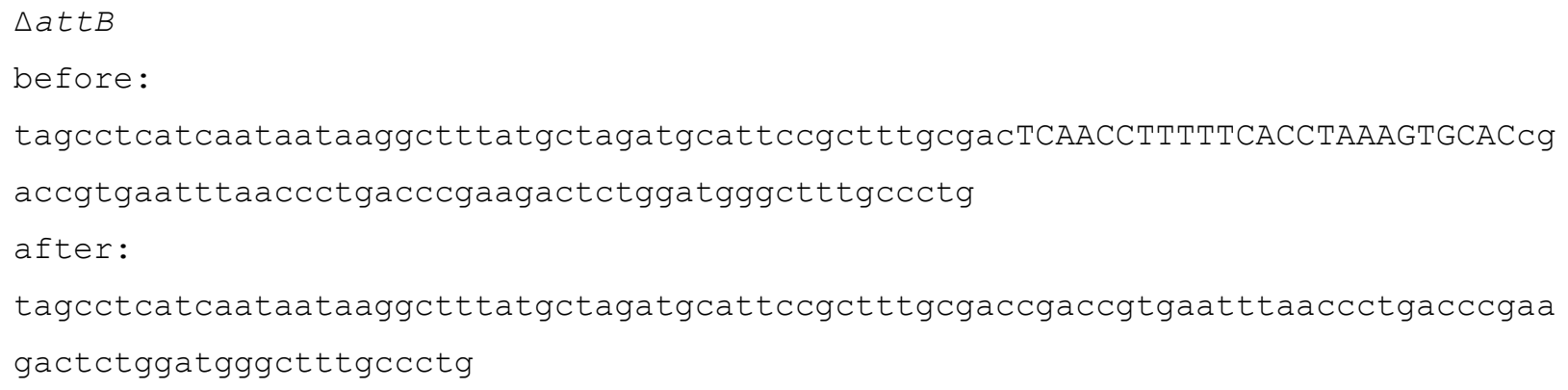


$\Delta$ tus

before:

ATGGCGCGTTACGATCTCGTAGACCGACTCAACACTACCT'

GCACTGATTAATCGGGATAATGGCGCGGGCGTGCCGGACGTTGGTGAGTT

after:

ATGGCGCGTTACGATCTCGTAGACCGACTCAACACTACCTTtcgccagatCTGATTAATCGGGATAATGGCGCGGGC

GTGCCGGACGTTGGTGAGTT

Table S3. The modifications to the genome sequence of DGF-298W $\Delta 100:$ :rev $\Delta 234:: S C$ to develop RGF008 and its $\triangle$ recAX derivative.

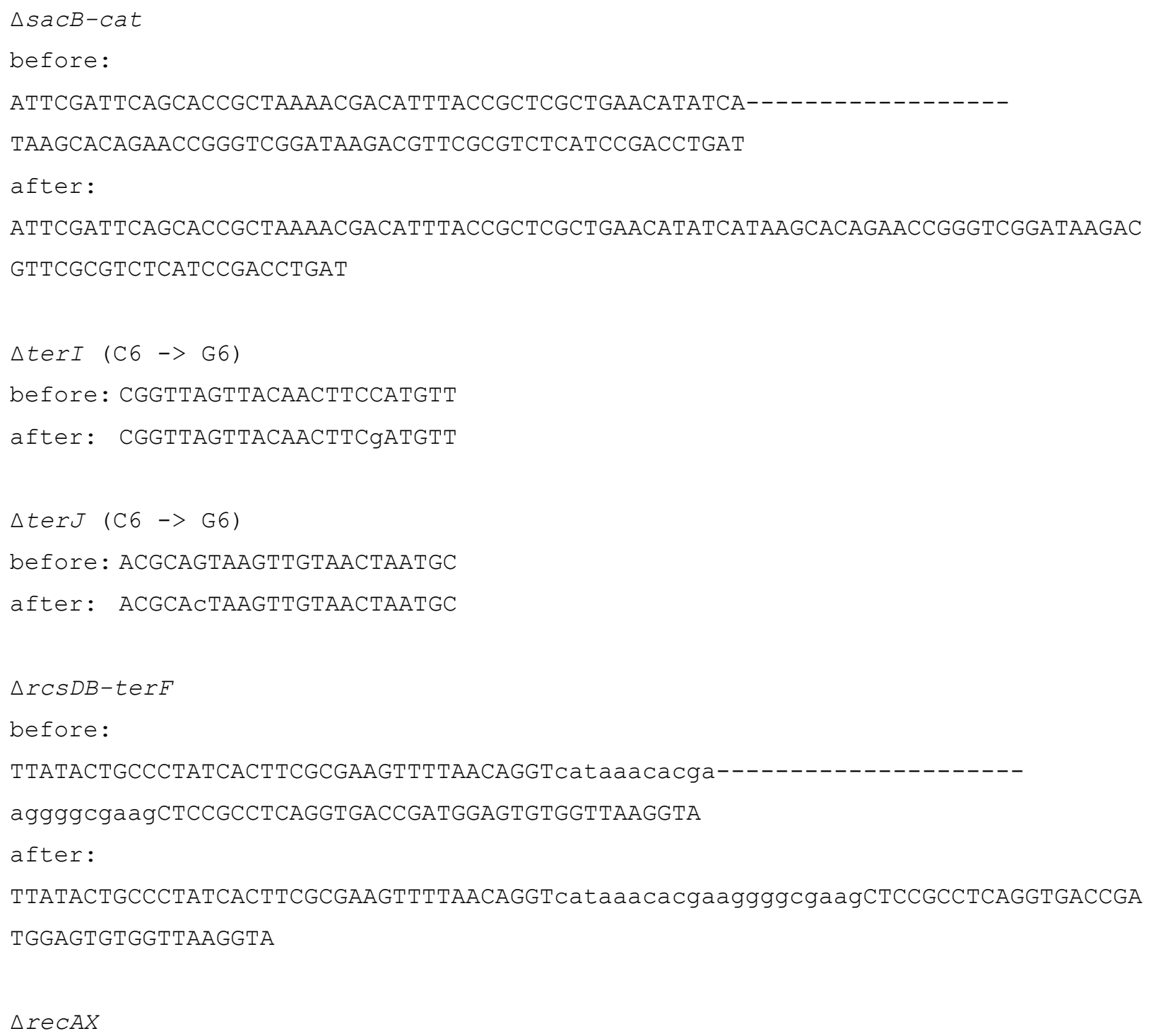


before:

CCACTGCCCGCGGTGAAGGCATTACCCGGCGGGAATGCTTCAGCGGCGAC----------------

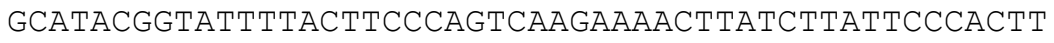

after:

CCACTGCCCGCGGTGAAGGCATTACCCGGCGGGAATGCTTCAGCGGCGACCGTGATGCGGTGCGTCGTACGCATACG

GTATTTTAСтTCCCAGTCAAGAАAАСтTAтCTTATTCCСАСтT

Table S4. The sequences of the homology arms used in the lambda Red recombination experiments. Lower-case nucleotides indicate that they were not always used.

a $0.5^{\prime}$

Fwd: GCTTTTTAGTCCAGCGGTGCGTTAATCGGCAGCTCCCCCAAAGTTAAGGT

Rev: GCCAACTTACTGATTTAGTGTATGATGGTGTTTTTGAGGTGCTCCA

a $2.6^{\prime}$

Fwd: TGgTCGTGGTGGTGATtGCACTACTGACGATTTACGGGGCGTTGGTGTAA

Rev: CtATCtgttTAAAgAgAAAACGCCAAAgCCGTAAAAgCGCATtAA

Q $3.7^{\prime}$

Fwd: GTTTATGGCGGCAGTCGTCGGCACGCTTGTTGGGCTGGCAGCGGTTGCTT

Rev: AGCGCCCCCATACGTTGGTTCTGCAACCAGGCGACACCTTTGTCAA

(2) $4.8^{\prime}$

Fwd: GCCGTCAATGAAATGGATGTTAAGGCATCCAGACGTCTAAATCAATCAGG

Rev: ctAgtATCGCATGtTtCGACCTGCAAGAAAgTGCTCTTCGCATAAA

(a) $4.9^{\prime}$

Fwd: ATCTGCCATTAACTCCACCAATTCTCCGCGAGCGATTTCTTTGTCGATCA

Rev: CAGCTTtgcctGAgTGGCAACGGGATtGCGTGTTTGTCCGACTACA

a. $5.7^{\prime}$

Fwd: GTTACGTAAGATCTTATCATTCTCCCACCAAAAAATTATCTTAATGTAAC

Rev: AgtAgAtAAgAtTGATCTTCGTtgAtAgAAтtTAстTACACCAGCT

(2) $9.3^{\prime}$

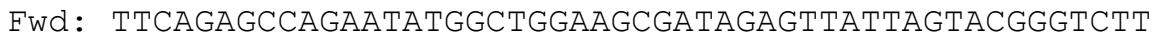

Rev: CATCGGCTTCACCAACTTCGACTGGGGTTCCGATTTAGGGGATGAC 
Q13.1'

Fwd: AAtCAgCCAGCTTGCCCAGGTGTGTCTGAgGTCATGGAAACGGAAATCTT

Rev: CGCGTTCTTCAATTGCGTTTTGTAGGCTGTCAGGGATACTGTCCCA

a $16.2^{\prime}$

Fwd: gcCGACTACGCTCTGCATTTGCCGAAAAGGGGATTGTTGTCGCAGCATAA

Rev: TCTGGCGTAGCTATACCCCTATTCTATATCCTTAAAGGACTCTGTT

Q18.9'

Fwd: CTCTCTGGGCGGCTCCTGCAACGGTAAATGTCGAAGTACTGCAAGACAAACTCGACCATC

Rev: CGCCGCCGCGCAGAGTGATTAACATACCGTGATTATCGGGTAAAAAGGCCAGTGCCCAGG

(a) $24.6^{\prime}$

Fwd: tcgctttAAAACATATCATGAAACTGGGTATGTTTTGTCTGCCTGCTC

Rev: ttaAAATTCATCGAATGGCATCCTTGCTAACCAACAATGCAAAATAGGC

(a $41.4^{\prime}$

Fwd: tacCGGTGCTGTCTTTGGGGGCGAACTGACTCTTGTGCAATTGCAATAA

Rev: tTTTATAGATGGTTTCTGGCGACCTGCACAGGAGTACGGTGATTTTTAA

(a $46.9^{\prime}$

Fwd: AACGCCTTATCCGTCCTACGGGGTGGTGTTGTTCGTATAAGGCGTA

Rev: TAACGCCGTGCAGGACGTTTATCTCGATAGCAAAATTACTATCGCCTGA

Q $50.7^{\prime}$

Fwd: aaTGAGTCGCATCATTACCGATTCATATCAATAATCTATTTTTGTAGCT

Rev: tccgTGCCCAATGTATGCGTTGCAACGCAGTGAAAATTCCTCTGA

a $52.5^{\prime}$

Fwd: CTGCGGTGCTGGGTATTATtGTCACGATACTGTCATTTCGTCGGGGTTAA

Rev: ACTGTTAGTCGCGATTATCCTGATGCTGGTCTGGCTGGTTCGTTAA 


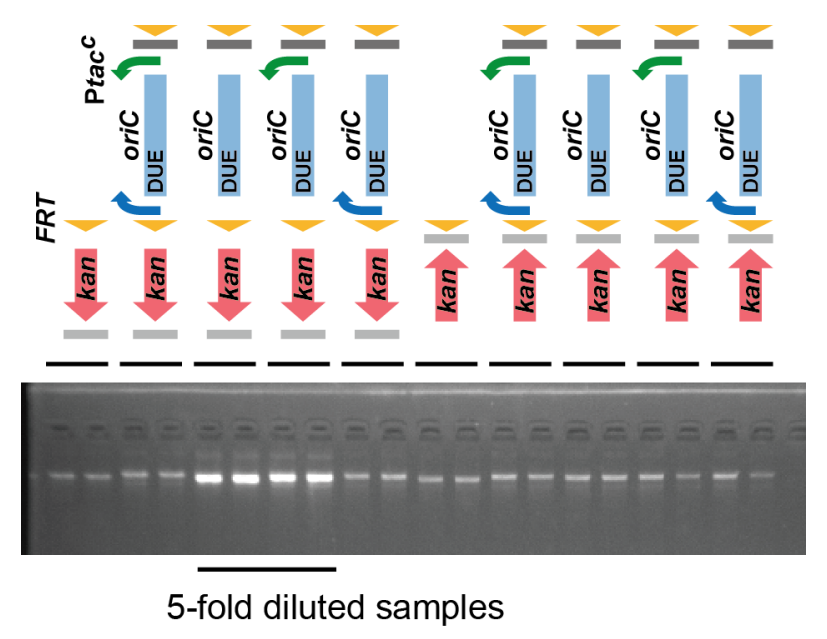

Figure S1. Yields of mini-pSa plasmids carrying the indicated cassettes. Plasmids prepared by miniprep from two different colonies were analysed by agarose gel electrophoresis. The oriC-less plasmids were made by trimming the oriC sequence flanked by the $F R T$ sites by using flippase. Ptac ${ }^{C}$ is a constitutive variant of Ptac lacking the operator sequence. For high-copy-number variants, their DNA solutions were diluted and analysed by agarose gel electrophoresis. The rightmost cassette is used as RCR ori with or without the FRT sites.

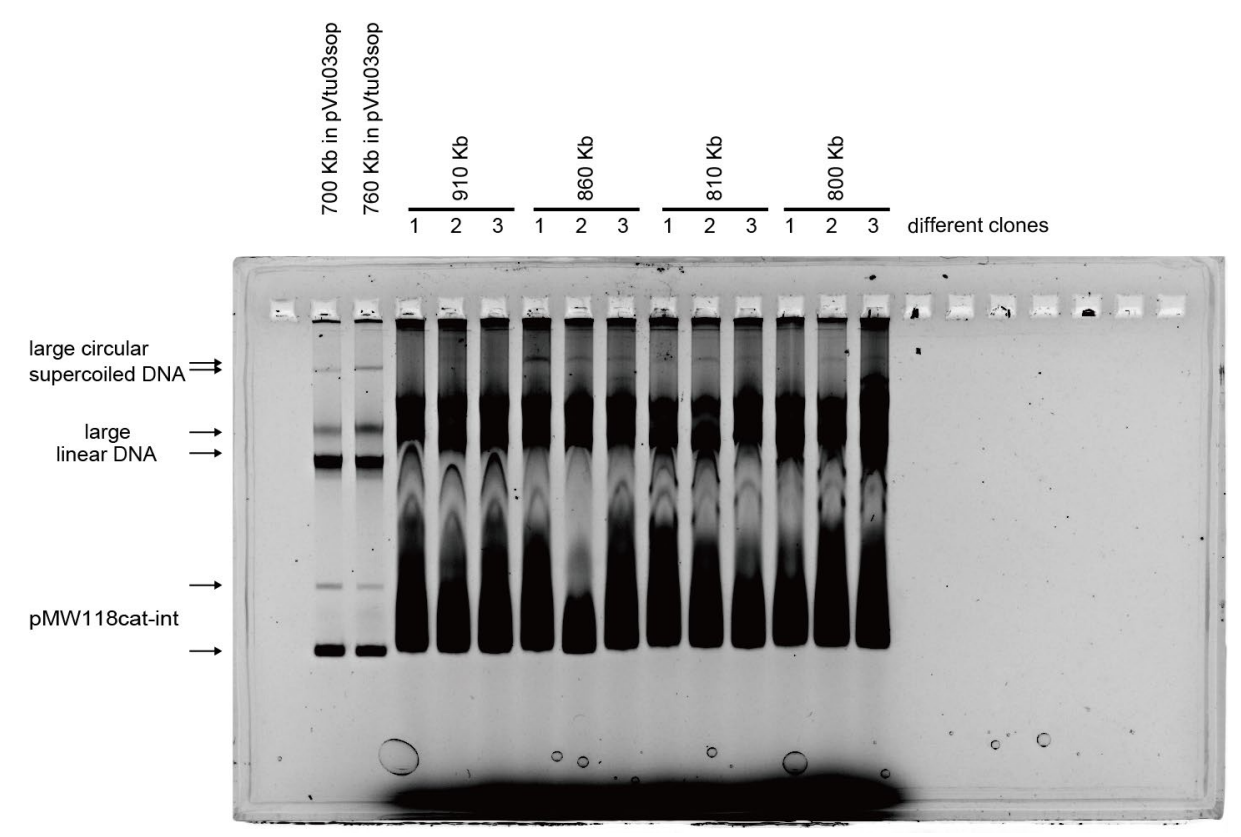

Figure S2. Rapid BAC purification of $800 \mathrm{~kb}$ to $900 \mathrm{~kb}$ plasmids from three different colonies. pMegaBAC1 was used as vector. Apparently, vast amounts of contaminating molecules prevented proper resolution of plasmids and estimation of the plasmid sizes by agarose gel electrophoresis. 
A

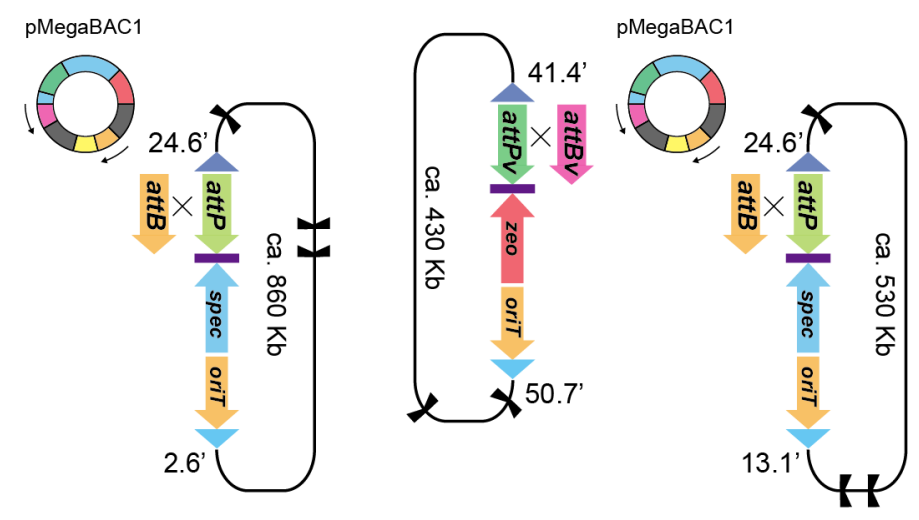

B

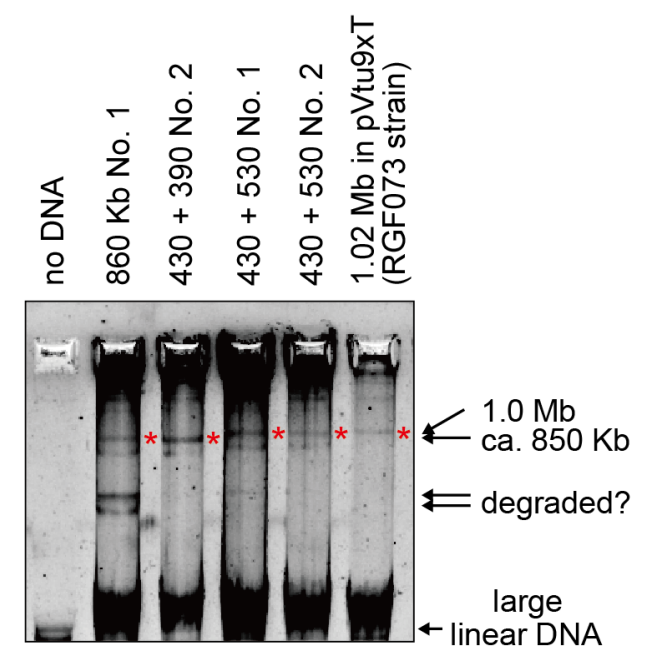

Figure S3. Construction of sub-megabase-sized plasmids by using pMegaBAC1. (A) Procedures for cloning one to two sub-megabase-sized genome regions into a single pMegaBAC1 vector. The ter sequences in the cloned regions are indicated with black bars. (B) Agarose gel electrophoresis analysis of the supercoiled form of megabase-sized plasmids amplified by RCR by using a $0.5 \%$ agarose gel with TBE buffer. The red stars indicate the desired bands. The agarose plugs used to extract the DNA templates for RCR were made by using the in-gel cell-lysis step with lysozyme. 